\title{
Stimulation of osteoblast differentiation with guided ultrasound waves
}

\author{
James Macione ${ }^{1 *}$, Daniel Long ${ }^{1}$, Sterling Nesbitt ${ }^{1}$, Scott Wentzell ${ }^{1}$, Hiroki Yokota ${ }^{2}$, Vaibhav Pandit ${ }^{1}$ \\ and Shiva Kotha'
}

\begin{abstract}
Background: Ultrasound induces mechanical vibration and heat, causing differentiation and proliferation in osteoblasts. All known in vitro evaluations of ultrasound are, however, performed with longitudinal ultrasound waves. We addressed a question: Do other forms of ultrasound waves, such as guided waves (longitudinal and guided flexural) transduced at a remote location, enhance differentiation of osteoblast cells?
\end{abstract}

Methods: In this study, we employed guided Lamb waves that were induced in a borosilicate glass slide (cortical bone mimic). An average energy of $10-30 \mathrm{~mW} / \mathrm{cm}^{2}$ for 20 min per day was applied to MC3T3 osteoblast-like cells, which were placed 30-75 $\mathrm{mm}$ distant from the transducer.

Results: The result revealed that guided waves significantly stimulated the differentiation and mineralization of MC3T3 cells. In particular, guided waves elevated mRNA expression levels of bone formation-related genes such as alkaline phosphatase, osteopontin, osteocalcin, osteoprotegerin, and bone sialoprotein on days 8 and 16 . In addition, the amount of mineralization found via Alizarin red staining was increased by $157 \%(p=0.034)$. The amount of mineralization was found to be independent of distance from the transducer $(p=0.967)$.

Conclusion: We demonstrate herein that ultrasound in a form of guided Lamb waves is capable of inducing osteoblast differentiation in vitro, and it may enable the stimulation of osteoblasts in vivo over a distance from the site of ultrasound application.

\section{Background}

Ultrasound can be used as a diagnostic and therapeutic tool in musculoskeletal diseases and disorders. As a diagnostic tool, for instance, it is utilized to evaluate mechanical properties of bone in which guided waves are induced into the cortical bone [1-4]. The induced waves are called "guided" as the cortex has a longitudinal speed of sound (SOS) and impedance that are double the surrounding tissue and internal trabecular bone [5]. As the guided wave propagates along the thin shelled cortex, particle motion-based flexing and extending occurs, heat is generated, and a small amount of mechanical energy, the leaky wave, is transmitted into the boundary tissues [6]. The concept of guided waves in the bone is not novel; commercial ultrasonic bone

\footnotetext{
* Correspondence: james.macione@gmail.com

'Department of Biomedical Engineering, Rensselaer Polytechnic Institute, Troy, NY 12180, USA

Full list of author information is available at the end of the article
}

density devices utilize the guided waves over ranges from 5 to $8 \mathrm{~cm}[1,2]$.

As a therapeutic tool, ultrasound in a form of longitudinal waves has been used to enhance bone growth and repair fracture including nonunion [7-12]. For example, ultrasound stimulates pre-osteoblasts to differentiate into osteoblasts [13-15]. The gold standard ultrasound signal is a $1.5-\mathrm{MHz}$ longitudinal wave with a $20 \%$ duty cycle that yields a spatial average, temporal average (SATA) intensity of $30 \mathrm{~mW} / \mathrm{cm}^{2}$, and an FDA-approved power level [16]. The bone is treated via longitudinal waves, allowing waves to travel through soft tissue and ultimately reach the outer cortical surface. Sometimes, the waves can have conversion to shear and longitudinal components [16], even when induced at an angle to try to isolate these wave components [17].

The ultrasonic stimulation of the bone would serve a potential way to offset osteoporosis, but yet, it is only utilized for bone union and in culture $[16,17]$. The reason for the limitations is because it has limited efficacy 
in stimulation within intact bone in vivo. Yet, these bone cells are sensitive to mechanical forces as the lack of this stimulation is one of the major causes of low bone mass. In an effort to understand why ultrasound can stimulate cells in culture, but not in vivo, some have started to examine different types of ultrasonic effects found in culture wells [18-20]. Others have evaluated different types of mechanical effects in culture such as frequency mixing [21] or variations of the applied frequency and intensity [22].

To our knowledge, all known in vitro evaluations of ultrasound stimulation of bone cells have been initiated with longitudinal waves passing through a medium prior to reaching the bone cells. This is not necessarily a configuration that will model what can occur in vivo. In this study, we explore the use of guided waves through a stimulated cortex in an effort to stimulate in vitro cells with guided Lamb waves.

\section{Materials and methods}

Guided wave speed of sound and wavelength in glass and bone

The velocity of the different types of guided waves is dependent on the properties of the bone which include the thickness and longitudinal and transverse speed of sound. In order to compare the properties of flexural guided waves in glass and bone, Lamb's equations were solved without fluid/tissue loading conditions using numerical methods on formulas with known constants from Dodd et al. [4] with methods from Rose [23] (Fig. 1). The phase velocity dispersion curve of the two lowest order antisymmetric and symmetric modes $\left(S_{0}, S_{1}, A_{0}, A_{1}\right)$ for bone and borasilicate glass is shown in Fig. 1. In both dispersion curves, at a frequency*diameter (diameter of the cortical thickness) product of $1.2 \mathrm{MHz} \mathrm{mm}$, there are two possible modes, the first-order antisymmetric $\left(A_{0}\right)$ and symmetric $\left(S_{0}\right)$.

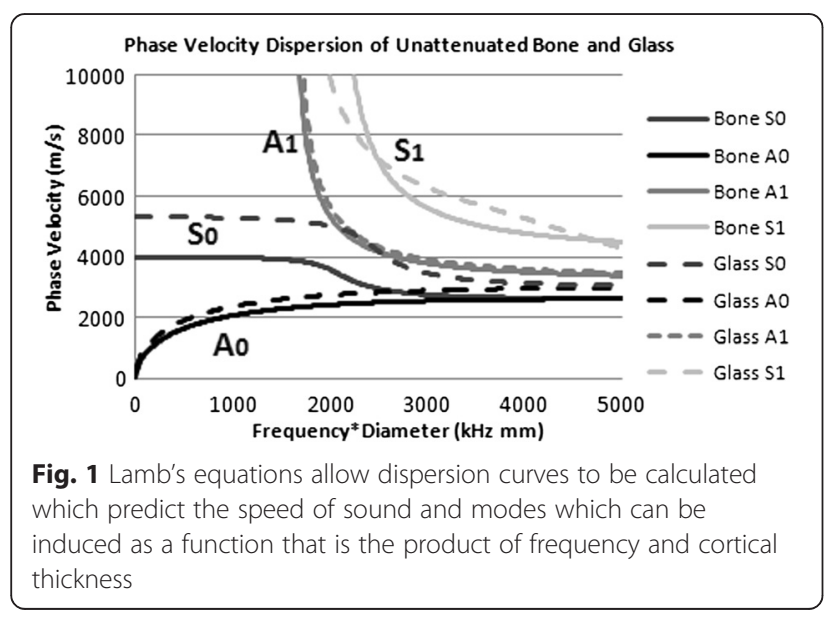

\section{Cell culture and mineralization}

Mouse osteoblast-like cells (MC3T3-E1 subclone 14, passage 7) were cultured in a solution of $\alpha$-Modified Essential Medium (Invitrogen $\alpha$-MEM 11900-024 with $2.2 \mathrm{~g}$ of $\mathrm{NaHCO}_{3}$ ), $10 \%$ fetal bovine serum, and $100 \mu \mathrm{L} / \mathrm{mL}$ of penicillin/streptomycin [24]. The cells were grown in a T-75 culture flask until confluence was reached, at which point they were seeded into 8-well Lab-Tek chamber slides (catalog: 154534/glass, Thermofisher Scientific, Waltham, MA, USA) at a density of 4445 cells per $0.8 \mathrm{~cm}^{2}$. Differentiation was induced after confluence by adding mineralizing media. This mineralizing media is the aforementioned solution, supplemented with $10 \mathrm{mM} \beta$ glycerophosphate and $50 \mu \mathrm{g} / \mathrm{mL}$ of ascorbic acid. The media was changed the day after seeding and every 2 days thereafter. Note that when the media was changed, a heating pad was used to keep the cells warm $\left(37^{\circ} \mathrm{C}\right)$ since they were on a large glass sheet $(75 \times 100 \mathrm{~mm})$ which induced a large, rapid drop in temperature $\left(4-5{ }^{\circ} \mathrm{C}\right.$ in $\left.20 \mathrm{~s}\right)$ when placed in contact with the metal working surface of the biosafety cabinet.

\section{Slide preparation}

Prior to attachment of the cell chambers, each borosilicate slide (product ID: 260230, Ted Pella, Redding, CA, USA) was washed and rinsed five times with soap/water and then rinsed for additional five times with deionized water. After drying, the slides were acid etched in piranha solution (3:1 ratio of concentrated sulfuric acid to $30 \%$ hydrogen peroxide) in order to remove organic contaminants. The slides were then rinsed for additional five times in water before drying for 7 days in a biosafety cabinet under UV light (to assist with sterilization). After drying was complete, a Lab-Tek chamber slide was removed (by prying) from its glass slide and reseated onto the borosilicate glass. In order to prevent leaks, the edges of the chamber slides were reinforced by adding a small amount of PDMS (Sylgard 184 Kit, Dow Corning, Midland, Michigan) in 10:1 ratio (monomeractivator), which was cured overnight at $50{ }^{\circ} \mathrm{C}$.

\section{Distance and attenuation effects}

The use of chamber slides allows the effects of attenuation to be evaluated. The Lab-Tek chamber slides have a $2 \times 4$ well configuration in which each of the four groups of two are at different distances $(30,45,60$, and $75 \mathrm{~mm}$ ) from the transducer. This $15-\mathrm{mm}$ distance is large enough that attenuation-based changes in intensity can be measured at the four locations and then correlated with the amount of mineralization that occurs.

\section{Ultrasonic configuration}

Pulsed, guided waves were created so they would utilize the glass slide as a waveguide. An electronic timing 
circuit was used to pulse a $1-\mathrm{MHz}$ ultrasound power amplifier at $1 \mathrm{KHz}$ (SATA $30 \mathrm{~mW}$ ) with $20 \%$ duty cycle. The use of the pulsing circuitry allows comparison with other studies, as well as gives the slower modes (such as flexural $A_{0}$ ) time to propagate though the plate (without being overtaken by faster modes), and also helps reduce the amount of standing waves. The borosilicate glass slide $\left(75 \times 10 \times 1.2 \mathrm{~mm}^{3}\right)$ was configured to act as a waveguide (Fig. 2) as the top was bound by cell media $\left(V_{\mathrm{L}}=5600\right.$ vs. $1500 \mathrm{~m} / \mathrm{s}$ ) while the bottom was isolated from the metal trays of the incubator with a small sheet of 25-mm-thick expanded polystyrene. Thus, the pulsed guided waves would travel through the glass and leak into the cell chambers. Other losses would be governed by only attenuation and destructive interference.

\section{Validating guided and leaky waves}

The presence of the guided waves as well as power of the leaky waves was validated by speed of sound measurements. A hydrophone was placed into a well filled with water. This signal, along with the start of the $1 \mathrm{kHz}$ pulse was recorded by a $400-\mathrm{MHz}$ data acquisition device (DR200 with PX14400, Signatec, Newport Beach, CA, USA). The first wave to arrive has a SOS of approximately $4800 \mathrm{~m} / \mathrm{s}$ with the second group(s) at $2200 \mathrm{~m} / \mathrm{s}$, with some signals arriving later due to reflection (R) from the back of the glass plate (Fig. 3a). The first group of waves are denoted $S_{0}{ }^{*}$, which indicates that it starts with the faster $S_{0}$ waves but afterward becomes a combination of both modes. In the same manner, the slowest waves at the end of each pulsing cycle are denoted $A_{0}{ }^{*}$. The waves between the start of $A_{0}{ }^{*}$ and end of $S_{0}{ }^{*}$ are a combination/superposition of both modes.

\section{Leaky lamb wave force}

The amount of energy from the leaky waves used to stimulate the cells was measured with the HNR-1000 hydrophone after each well was filled with media. The hydrophone was placed in each well and slightly angled toward the transducer in order to find the peak intensity.
The maximum peak voltage of the waveform over $30 \mathrm{~s}$ was converted to a power level $\left(\mathrm{W} / \mathrm{cm}^{2}\right)$ from the hydrophone calibration curve $\left(\mathrm{V}^{2} \mathrm{~cm}^{2} / \mathrm{W}\right)$ at $1 \mathrm{MHz}$ and was found to vary from 30 to $10 \mathrm{~mW} / \mathrm{cm}^{2}$ SATA from the well closest to the well furthest from the transducer.

\section{Alizarin red staining for calcium}

Alizarin red staining was used to image and quantify mineralization [21]. Samples were fixed using $70 \%$ ethanol for $1 \mathrm{~h}$ before mixing with $40 \mathrm{mM}$ alizarin red at $\mathrm{pH}$ 4.2 for 10 min and finally washed with tap water before being allowed to dry. Mineralization of the samples was done via microscopy. The amount of calcium was then quantified by destaining with a solution of $10 \%$ cetylpyridinium chloride and $10 \mathrm{mM}$ sodium phosphate for $15 \mathrm{~min}$ at room temperature. The absorbance of each sample was determined at $562 \mathrm{~nm}$ and calibrated to standards of alizarin red diluted with $10 \mathrm{mM}$ sodium phosphate containing $10 \%$ cetylpyridinium chloride. In order to determine statistical relevance, a post hoc Tukey and paired $t$ tests Minitab 15 (State College, PA) were used for ANOVA analysis. Statistical significance is considered any $p<0.05$. A total of eight glass slides were used to access mineralization, this yielding eight wells at each distance (two wells at each distance on one plate) from the transducer for both the control and ultrasound stimulated samples.

\section{Quantitative polymerase chain reaction}

Quantitative PCR (qPCR) was performed to analyze mRNA expression levels that indicate cellular differentiation. Total RNA was extracted with TRIzol LS reagent (Life Technologies, Carlsbad, CA, USA) and treated with deoxyribonuclease. After DNase treatment, reverse-transcription was conducted using oligo-p(dT) primers. PCR amplification was performed on Lightcycler 480 (Roche, Penzberg, Germany) using SYBR green master mix (Qiagen, Venlo, Netherlands) and PCR primers in Table 1. Reactions were carried out in total volumes of $20 \mu \mathrm{L}$ and included $0.3 \mu \mathrm{M}$ of each primer (forward and reverse) and $0.5 \mu \mathrm{L}$ of diluted

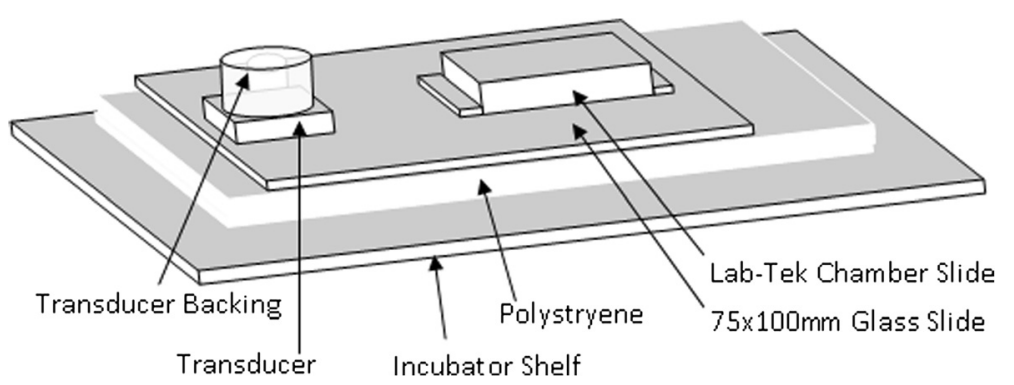

Fig. 2 The cells are grown on an acid etched, optical grade borosilicate slide within the Lab-Tek Chamber slide. The distance between the chamber slide and the nearest edge of the transducer is approximately $30 \mathrm{~mm}$. Lamb waves propagate through the glass which extend/flex it and allow ultrasound to "leak" out to stimulate the cells 

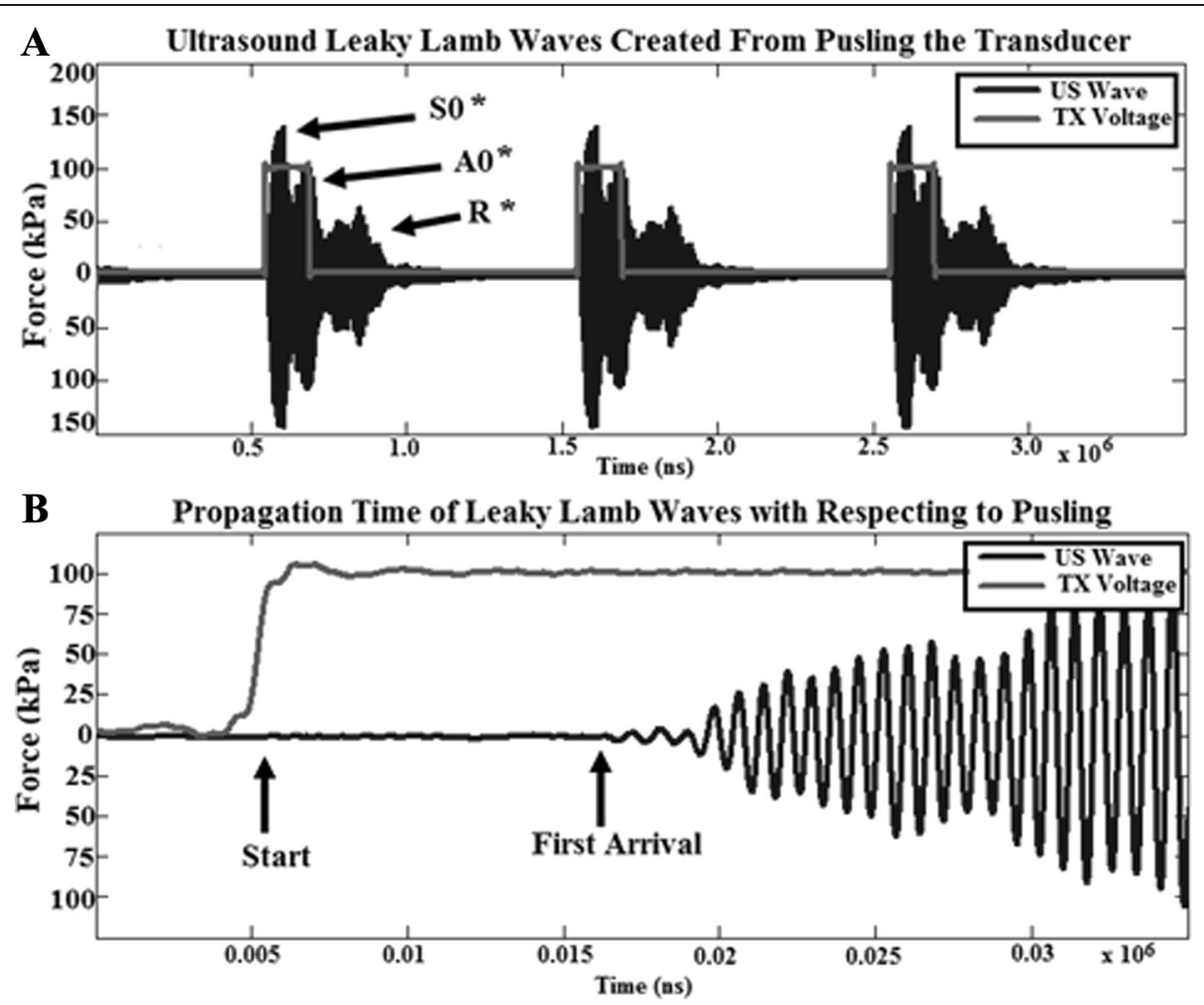

Fig. 3 a A $1 \mathrm{kHz}$ drive signal with $20 \%$ duty cycle plotted with the hydrophone recording from the chamber slide on the borosilicate plate. The asterisk indicates that the $S_{0}$ and $A_{0}$ modes are mixed since each transducer pulse will create both modes which have different SOS. $\mathbf{b}$ The first arrival can be used to compute the SOS in order to verify the presence of Lamb waves. In this case, over $75 \mathrm{~mm}$, a SOS of $4900 \mathrm{~m} / \mathrm{s}$ is determined which corresponds to the lowest order symmetrical Lamb mode $\left(S_{0}\right)$

cDNA template containing 1000 ng of cDNA. Statistical significance was determined with the MATLAB (Mathworks, Natick, MA, USA) ANOVA function. Statistical significance is considered any $p<0.05$. A total of eight glass slides (four control, four stimulated) were used for PCR, but due to the expense of running reactions, the wells on each were mixed to form a single sample.

\section{Results}

Alizarin red staining demonstrated that both the control and ultrasound-stimulated sample had nodule formation.
However, the mineral deposition was increased in the samples stimulated by guided waves (Fig. $4 \mathrm{a}-\mathrm{c}$ ). Quantification of mineralization through a process of Alizarin red destaining revealed that an average of $157 \%$ more mineralization was achieved in the stimulated samples $(p=0.034)$.

The increase in mineralization was not dependent on the distance from the transducer $(p=0.967)$. This distance from the transducer is related to the amount of leaky wave energy the cells would experience, ranging from $30 \mathrm{~mW} / \mathrm{cm}^{2}$ at $30 \mathrm{~mm}$ to $10 \mathrm{~mW} / \mathrm{cm}^{2}$ at $75 \mathrm{~mm}$. There was a slightly higher mineralization at the $30-\mathrm{mm}$

Table 1 Primers for qPCR in this study

\begin{tabular}{lll}
\hline Gene & Forward primer & Reverse primer \\
\hline GAPDH & AACGACCCCTTCATTGAC & TCCACGACATACTCAGCAC \\
OPN & GATCAGGACAACAACGGAAAGG & CTTGTGGCTGTGAAACTTGTGG \\
OCN & AGGGAGGATCAAGTCCCG & GAACAGACTCCGGCGCTA \\
BSP & TGTCTGCTGAAACCCGTTC & GGGGTCTTAAGTACCGGC \\
ALP & GTTGCCAAGCTGGGAAGAACAC & CCCACCCCGCTATTCCAAAC \\
OPG & CGAGGACCACAATGAACAAGTG & TITAGGTAGGTGCCAGGAGCA
\end{tabular}

GAPDH glyceraldehyde-3-phosphate dehydrogenase, $O P N$ osteopontin, $O C N$ osteocalcin, $B S P$ bone sialoprotein, $A L P$ alkaline phosphatase, and $O P G$ osteoprotegerin 
$\mathbf{A}$

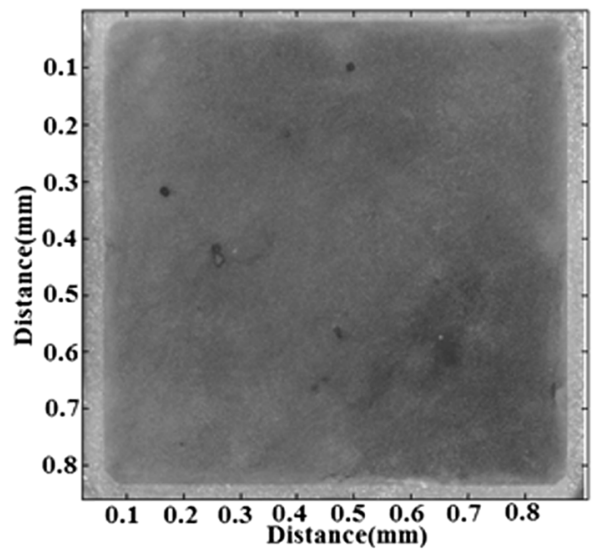

B

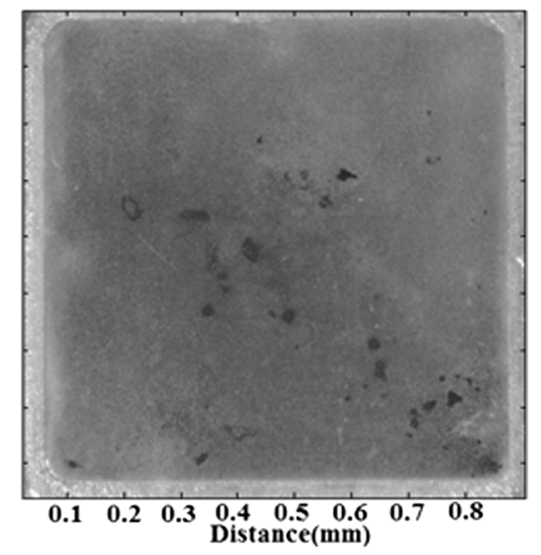

C

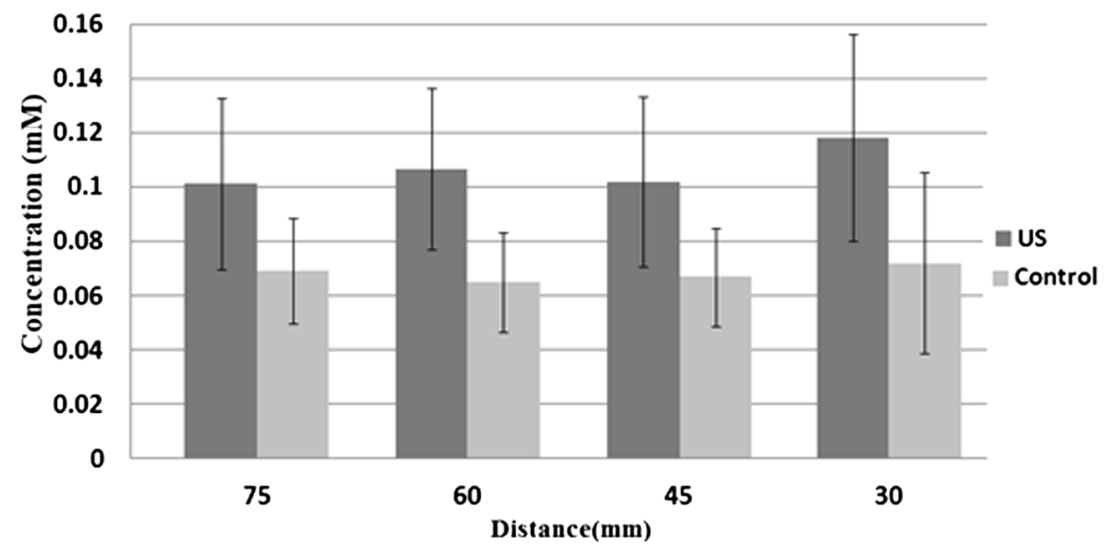

Fig. 4 a, b Representative images from alizarin red staining of both the control (a) and guided wave samples (b) at day 25. Quantifying the mineralizaiton (c) demonstrates that samples stimulated with guided waves have an average of $157 \%$ more calcium, an effect which was independent of distance

sample, but the difference among the samples was not statistically significant.

\section{Guided wave-induced elevation of relative mRNA expression levels}

The relative mRNA expression levels of the five selected genes were examined on days 8 and 16 in the presence and absence of ultrasound in guided waves. At both time points, the relative expression levels were significantly greater than 1, indicating that differentiation of osteoblasts was accelerated in the ultrasound-stimulated samples (Table 2). At day 8, the relative gene levels for alkaline phosphatase (ALP), osteopontin (OPN), osteocalcin (OCN), osteoprotegerin (OPG), and bone sialoprotein (BSP) increased 2.5-6.8-fold. The relative gene expression became larger at day 16, except for ALP and OPG. For instance, the relative mRNA level of BSP was $6.8 \pm 1.1$ (mean \pm s.d.; $p<0.01$ ) on day 8 and $10.2 \pm 4.2$ $(p<0.01)$ on day 16. The decrease in ALP is expected since it is considered as an early marker for osteoblast differentiation [25].

\section{Discussion}

Guided waves travel within cortical bone and can have longitudinal and Lamb wave components. The guided waves will leak energy into tissue boundaries and in the case of Lamb waves also can create very small amounts of flexion from out-of-plane particle motion. In this study, we created an acoustic representation of cortical bone with glass (Fig. 2) and then induced 1.2-MHz-mm guided flexural waves (Fig. 1) through a $30-\mathrm{mm}$ path length (Fig. 3) to produce a leaky wave SATA of 10 to $30 \mathrm{~mW} / \mathrm{cm}^{2}$. The cells were thus stimulated by leaky

Table 2 Fold change in relative mRNA levels by guided waves

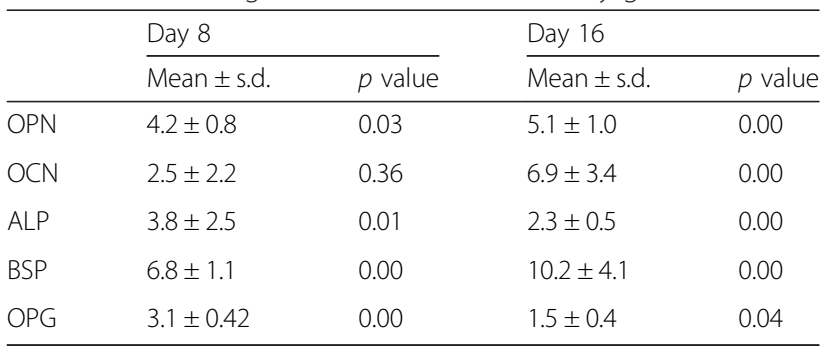


energy from the flexural waves which originate from the plate on which they reside as well as any small amounts of flexion which may occur. This is a different mechanism from the current standard of ultrasonic stimulation, in which only those cells directly within the field of the transducer are stimulated via the mechanism of longitudinal waves.

The guided waves were found to stimulate differentiation and mineralization in osteoblasts. Relative mRNA expression levels of the selected bone-linked markers increased three- to fivefold in 8 days of the ultrasound stimulation (Table 2). The mRNA levels stayed elevated on day 16, thus showing a continuous response to treatment. The guided waves also induced more mineralization, which could be seen in the form of nodules (Fig. $4 \mathrm{~b}, \mathrm{~d}$ ) which translated to greater calcium concentration (Fig. 4e). This increased mineralization was not found to be dependent on the distance of the sample to the transducer even though the leaky power from the Lamb waves was attenuated (30 to $10 \mathrm{~mW} / \mathrm{cm}^{2}$ ).

There was no dependence on the amount of mineralization with the distance from the transducer (leaky wave power). It is likely that the difference in intensity levels from 10 to $30 \mathrm{~mW} / \mathrm{cm}^{2}$ was not large enough to create a statically significant response. The results are consistent with a previous study that found that the cells stimulated with smaller $\left(20 \mathrm{~mW} / \mathrm{cm}^{2}\right)$ range of power level show only a small difference in response [22].

There were limitations to this study which were related to the selection of ultrasonic parameters. Ultrasound values of the driving frequency, power level, and pulse repetition frequency were selected by what others have done with longitudinal waves, and no attempt was made to optimize them for guided waves. For example, the pulsing frequency will alter the superposition (destructive interference of slower modes with faster) of the bulk of the pulses and even alter the amount of standing waves. The thickness of the glass was also a limitation in that it needed to be thin enough to allow light microscopy at the expense of mode velocity.

\section{Conclusion}

In this study, a mechanism of generating an osteogenic response was evaluated in vitro. Pulsed guided waves were found to produce a positive response when compared to a control sample (unstimulated). The guided waves allow cells distant from the transducer to be stimulated by allowing the energy to travel through the bone. Further work would be needed to optimize ultrasonic parameters specific to guided waves. Also, more knowledge related to the physics of guided flexural waves would be of interest.

\section{Appendix}

\section{Calculation of the Lamb mode velocity}

The velocity of each of the Lamb modes in glass were computed from dispersion curves which are dependent on properties of the bone including the longitudinal $\left(V_{\mathrm{L}}\right)$ and transverse $\left(V_{\mathrm{s}}\right)$ SOS and $d$. The velocity dispersion of the Lamb waves are described with the characteristic Eqs. 1, 2, 3, 4, 5, and 6 [23]. The wave number $k_{\mathrm{SA}}$ is numerically equal to $\omega / V$, where $V$ is the phase velocity of the Lamb wave and $\omega$ is the angular frequency. The phase velocity $(V)$ is related to the $\lambda$ by the relationship

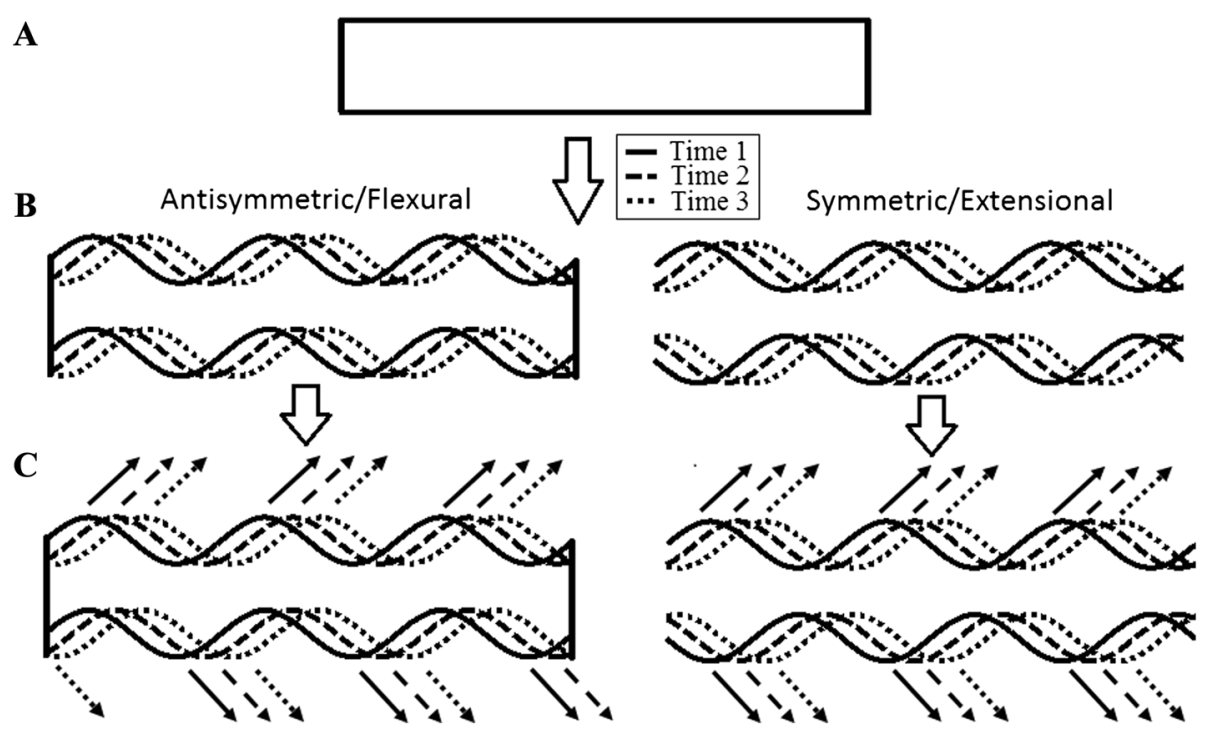

Fig. 5 a A schematic of a short section of a plate. $\mathbf{b}$ Exaggerated symmetric and antisymmetic flexural waves propagating through a plate at three closely related time points. c Both types of Lamb waves produce leaky waves which propagate out of the medium with respective directions. The symmetric half of the figure is from Lee and Kuo [6] 
$V=(\omega / 2 \pi) \lambda$ [23]. Thus, Eqs. 1, 2, 3, 4, 5, and 6 were solved numerically with Newton-Raphson technique to get the dispersion curves through glass:

$$
\begin{aligned}
& \left(k_{\mathrm{SA}}^{2}+T_{\mathrm{SA}}^{2}\right)^{2} \operatorname{coth}\left(L_{\mathrm{SA}} d\right)-4 k_{\mathrm{SA}}^{2} L_{\mathrm{SA}} T_{\mathrm{SA}} \operatorname{coth}\left(T_{\mathrm{SA}} d\right) \\
& \quad=0, \text { Symmetric }
\end{aligned}
$$

$$
\begin{aligned}
& \left(k_{\mathrm{SA}}^{2}+T_{\mathrm{SA}}^{2}\right)^{2} \tanh \left(L_{\mathrm{SA}} d\right)-4 k_{\mathrm{SA}}^{2} L_{\mathrm{SA}} T_{\mathrm{SA}} \tanh \left(T_{\mathrm{SA}} d\right) \\
& \quad=0, \text { Antisymmetric }
\end{aligned}
$$

where

$$
\begin{aligned}
& L_{\mathrm{SA}}=\sqrt{k_{\mathrm{SA}}{ }^{2}-\frac{w^{2}}{\mathrm{Vl}}} \\
& T_{\mathrm{SA}}=\sqrt{k_{\mathrm{SA}}{ }^{2}-\frac{w^{2}}{\mathrm{Vs}}} \\
& Q_{\mathrm{SA}}=\sqrt{k_{\mathrm{SA}}{ }^{2}-\frac{w^{2}}{\mathrm{Vl}}} \\
& K_{T}=\frac{w}{\mathrm{Vs}}
\end{aligned}
$$

As the Lamb wave propagates, at a level of particle motion, there is flexion around its midline and allows energy to escape (Fig. 5). The flexion and extension create different types of Lamb waves which are classified as antisymmetric and symmetric modes, respectively. The wave motions of the antisymmetic and symmetric waves have different propagation velocities, a property that depends on $d$ as well as the transducer frequency used to induce it.

\section{Competing interests}

The authors declare that they have no competing interests.

\section{Authors' contributions}

DL performed cellular experimentation, stimulated cells, and had theory toward experimental design. JM designed the experiments. HY had previous observations of mineralized cells distant to ultrasound application and was the senior advisor on cellular experimentation. SN provided acoustics and electronics theory. SK provided senior guidance toward acoustics theory and biology. VP did all PCR-related activities. SW provided acoustics theory and support toward experimental design. All authors read and approved the final manuscript.

\section{Acknowledgements}

Funding for this work was provided by NIH grants AG030637 (SK), AR052144 $(\mathrm{HY})$, and NSF grant 0846869 (SK).

\section{Author details}

'Department of Biomedical Engineering, Rensselaer Polytechnic Institute, Troy, NY 12180, USA. ²Department of Biomedical Engineering, Indiana University Purdue University Indianapolis, Indianapolis, IN 46202, USA.

\section{References}

1. Sarvazyan A, Tatarinov A, Egorov V, Airapetian S, Kurtenok V, Gatt Jr CJ. Application of the dual-frequency ultrasonometer for osteoporosis detection. Ultrasonics. 2009;49(3):331-7.

2. Prevrhal S, Fuerst T, Fan B, Njeh C, Hans D, Uffmann M, et al. Quantitative ultrasound of the tibia depends on both cortical density and thickness. Osteoporos Int. 2001;12(1):28-34.

3. Lee Kl, Yoon SW. Feasibility of bone assessment with leaky Lamb waves in bone phantoms and a bovine tibia. J Acoust Soc Am. 2004;115(6):3210-7.

4. Dodd SP, Cunningham JL, Miles AW, Gheduzzi S, Humphrey VF. Ultrasonic propagation in cortical bone mimics. Phys Med Biol. 2006;51(18):4635-47.

5. Laugier P, Haiat G. Bone quantitative ultrasound. New York: Springer; 2011.

6. Lee YC, Kuo SH. Leaky lamb waves of a piezoelectric plate subjected to conductive fluid loading: an experimental study. IEEE Trans Ultrason Ferroelectr Freq Control. 2006:53(9):1617-26

7. Suzuki A, Takayama T, Suzuki N, Sato M, Fukuda T, Ito K. Daily low-intensity pulsed ultrasound-mediated osteogenic differentiation in rat osteoblasts. Acta Biochim Biophys Sin (Shanghai). 2009;41(2):108-15.

8. Heckman JD, Ryaby JP, McCabe J, Frey JJ, Kilcoyne RF. Acceleration of tibial fracture-healing by non-invasive, low-intensity pulsed ultrasound. J Bone Joint Surg Am. 1994;76(1):26-34.

9. Wang SJ, Lewallen DG, Bolander ME, Chao EY, Ilstrup DM, Greenleaf JF. Low intensity ultrasound treatment increases strength in a rat femoral fracture model. J Orthop Res. 1994;12(1):40-7.

10. Unsworth J, Kaneez S, Harris S, Ridgway J, Fenwick S, Chenery D, et al. Pulsed low intensity ultrasound enhances mineralisation in preosteoblast cells. Ultrasound Med Biol. 2007;33(9):1468-74.

11. Nolte PA, van der Krans A, Patka P, Janssen IM, Ryaby JP, Albers GH. Lowintensity pulsed ultrasound in the treatment of nonunions. J Trauma. 2001;51(4):693-702.

12. Martinez de Albornoz P, Khanna A, Longo UG, Forriol F, Maffulli N. The evidence of low-intensity pulsed ultrasound for in vitro, animal and human fracture healing. Brit Med Bull. 2011;100(1):39-57.

13. Korstjens $C M$, Nolte PA, Burger EH, Albers GH, Semeins $C M$, Aartman $\|_{\text {, }}$ et al. Stimulation of bone cell differentiation by low-intensity ultrasound-a histomorphometric in vitro study. J Orthop Res. 2004;22(3):495-500.

14. Nolte PA, Klein-Nulend J, Albers GH, Marti RK, Semeins CM, Goei SW, et al. Low-intensity ultrasound stimulates endochondral ossification in vitro. J Orthop Res. 2001;19(2):301-7.

15. Wu S, Kawahara Y, Manabe T, Ogawa K, Matsumoto M, Sasaki A, et al. Low-intensity pulsed ultrasound accelerates osteoblast differentiation and promotes bone formation in an osteoporosis rat model. Pathobiology. 2009;76(3):99-107.

16. Parvizi J, Vegari D. Pulsed low-intensity ultrasound for fracture healing. Foot Ankle Clin. 2005;10(4):595-608.

17. Chung SL, Pounder NM, de Ana FJ, Qin L, Sui Leung K, Cheung WH. Fracture healing enhancement with Low intensity pulsed ultrasound at a critical application angle. Ultrasound in Med Biol. 2011;37(7):1120-33.

18. Leskinen JJ, Hynynen K. Study of factors affecting the magnitude and nature of ultrasound exposure with in vitro set-ups. Ultrasound Med Biol. 2012;38(5):777-94

19. Patel US, Ghorayeb SR, Yamashita Y, Atanda F, Walmsley AD, Scheven BA. Ultrasound field characterization and bioeffects in multiwell culture plates. J Ther Ultrasound. 2015;3:8.

20. Hensel K. Analysis of ultrasound fields in cell culture wells for in vitro ultrasound therapy experiments. Ultrasound Med Biol. 2011;37:10.

21. Uddin SMZ. Low-intensity amplitude modulated ultrasound increases osteoblastic mineralization. Cell Mol Bioeng. 2011;4(1):81-90.

22. Suzuki A, Takayama T, Suzuki N, Sato M, Fukuda T, Ito K. Daily low-intensity pulsed ultrasound-mediated osteogenic differentiation in rat osteoblasts. J Ther Ultrasound. 2014;2(1):1-10.

23. Rose JL. Ultrasonic waves in solid media. Cambridge: New York: Cambridge University Press; 1999. xvi, 454.

24. Tanaka SM, Li J, Duncan RL, Yokota H, Burr DB, Turner CH. Effects of broad frequency vibration on cultured osteoblasts. J Biomech. 2003;36(1):73-80.

25. Stein GS, Lian JB, Stein JL, Van Wijnen AJ, Montecino M. Transcriptiona control of osteoblast growth and differentiation. Physiol Rev. 1996;76(2):593-629. 